\title{
Causes of renal oligohydramnios: impact on prenatal counseling and postnatal outcome
}

\author{
Sebastian $\operatorname{Loos}^{1} \cdot$ Markus J. Kemper ${ }^{2}$
}

Received: 16 October 2017 /Revised: 22 October 2017 / Accepted: 24 October 2017 /Published online: 11 November 2017

(C) IPNA 2017

\begin{abstract}
The presence of renal oligohydramnios ( $\mathrm{ROH})$ in a fetus has been associated in the past with a poor prognosis for survival, although recent studies have shown that survival has improved considerably due to the advances in neonatology and pediatric nephrology. In an article recently published in Pediatric Nephrology, evaluation of a large series by Mehler and colleagues confirms the improved prognosis, showing a survival rate of 32 of $38(84 \%)$. In addition, only 12 of 35 (34\%) neonates required renal replacement therapy. In five of these 12 children the dialysis could be terminated after the neonatal period. This study has important implications on the decision-making process and counseling of families. While $37 \%$ of families of the study opted for termination of pregnancies, palliative care was chosen by $8 \%$ of the families, representing an important option when a decision cannot be made rapidly by affected families. A multidisciplinary approach is not only necessary in the active treatment of neonates with a history of ROH but also in antenatal counseling. In this regard future efforts should establish consensus on an ethical framework for the decision-making process in $\mathrm{ROH}$.
\end{abstract}

Keywords Renal oligohydramnios · Palliative care · Antenatal counseling $\cdot$ Multidisciplinary approach $\cdot$ Ethics

Sebastian Loos

s.loos@uke.de

1 University Children's Hospital, University Medical Center Hamburg-Eppendorf, Hamburg, Germany

2 Department of Pediatrics, AK Hamburg Nord, Asklepios Medical School, Hamburg, Germany

\section{Introduction}

Renal oligohydramnios (ROH) is caused by bilateral renal dysfunction of the fetus. Underlying pathologies most often are associated with one of the three entities: (1) bilateral renal agenesis and/or hypo-/dysplasia, (2) urinary tract obstruction or (3) autosomal recessive polycystic kidney disease (ARPKD). These diseases lead to a lower urinary output in the fetus and subsequently to less amniotic fluid after the first trimester when the production of urine by the fetus sets in, contributing significantly to the amniotic fluid volume [1].

A prenatal diagnosis of $\mathrm{ROH}$ is often made during the routine ultrasound surveillance of pregnancies [2]. In Germany, these are provided at weeks 10, 20 and 30 of gestation. ROH is defined by surrogate parameters of the amniotic fluid volume measured by ultrasound, such as the amniotic fluid index or single deepest pocket [1]. Although the ultrasound criteria for diagnosis are still a matter of debate, an amniotic fluid index of $<5 \mathrm{~cm}$ is widely used as a definition of $\mathrm{ROH}$ [3].

$\mathrm{ROH}$ can be associated with severe perinatal morbidity and postnatal mortality. The postnatal course is dominated by lung hypoplasia and chronic kidney disease (CKD) [1] Lung hypoplasia is one of the main causes of neonatal morbidity and mortality. In the past, these potential problems often led to termination of the pregnancy (TOP). Advances in treating respiratory failure and performing dialysis in even very small infants have tremendously improved survival and short- as well as long-term outcome in affected children. In this context, TOP is generally not longer recommended [1].

Several antenatal prognostic factors of children born after $\mathrm{ROH}$ have been evaluated to anticipate the postnatal course. These factors may have an important impact for 
prenatal counseling. However, postnatal kidney function is difficult to predict based on ultrasound studies, especially the long-term outcome $[4,5]$. Magnetic resonance imaging scans can provide additional information on fetal urinary tract abnormalities in some cases, but these have not been evaluated as a modality to predict postnatal outcome $[6,7]$. Therefore, some studies have focused on the assessment of biochemical markers in fetal urine or blood to predict the postnatal outcome of kidney function. These markers include urinary sodium, chloride and urinary and serum $\beta 2$-microglobulin and cystatin $C$ [1]. Unfortunately, none of these markers (alone or in combination) has sufficient sensitivity or specificity to predict postnatal outcome. In addition, sampling always bears the risk of an invasive procedure during pregnancy.

These risks also exist in prenatal interventions such as deobstructive procedures and amnioinfusion, the latter thought to prevent or ameliorate pulmonary hypoplasia; randomized controlled trials, however, are lacking [8]. De-obstructive procedures (vesico-/renoamniotic shunting, aspirations from bladder/kidney or vesico-/ureteostomies) have been performed in fetuses with urinary tract obstruction to improve pre- and postnatal kidney function $[9,10]$. In recent years most studies have focused on vesicioamniotic shunting. Again, however, prospective controlled trials are scarce, and those which have been conducted did not show a benefit [10]. Since clear prognostic parameters are lacking, no stratification for such interventions is possible to date.

Postnatal care has changed tremendously during the past decades due to improved ventilation and dialysis techniques. Under these conditions mortality, mostly due to pulmonary hypoplasia, ranges from 30 to $50 \%$ and up to $75 \%$ (Table 1) $[9,11-18]$. However, the high variation in mortality between studies is dependent on the decision regarding TOP and the co-morbidities of liveborn children, both factors which could be misleading in the interpretation of data (Table 1).
Lastly, to date, no studies on the underlying cause of $\mathrm{ROH}$ (e.g. ARPKD vs. urinary tract obstruction) and its impact on the decision against or for TOP have been published.

\section{Clinical impact of the study by Mehler et al. [19]}

In their retrospectively analyzed, well-described cohort of 103 pregnancies, Mehler et al. present all steps in the care of pregnant women with $\mathrm{ROH}$ and in newborns with a history of $\mathrm{ROH}$, from prenatal counseling based on the renal pathology to postnatal treatment and outcome [19]. The authors found that 38 of the 103 (37\%) pregnancies were terminated after prenatal counseling - unfortunately, often after counseling by obstetricians only. The decision for TOP was associated with the presence of other malformations, an earlier time-point of diagnosis of $\mathrm{ROH}$ and renal agenesis or kidney hypo-/dysplasia as the underlying cause of ROH. Of note, in the group with renal agenesis or kidney hypo-/dysplasia the diagnosis of $\mathrm{ROH}$ was made earlier than in the groups with ARPKD and obstructive uropathy, and the diagnosis of $\mathrm{ROH}$ was associated with the worst survival (30\%) in liveborn and actively treated children. Thus, the decision for TOP in this cohort seems to be based on known risk factors, such as onset of $\mathrm{ROH}$ and associated malformations $[11,13,14,16,17]$. However, the association of onset of ROH and outcome in liveborn children in general is debatable.

\section{Postnatal outcome after ROH}

Mehler et al. [19] report that invasive ventilation was necessary in 12 of the $38(32 \%)$ neonates who were actively treated; this also implies that $68 \%$ of neonates did not require invasive ventilator support despite a history of ROH. Of note, the rate of mechanical ventilation is hard to compare between different studies since it is strongly dependent on the decision of whether, and if so, to what extent to treat patients (Table 1).

Table 1 Postnatal outcome in liveborn children with renal oligohydramnios

\begin{tabular}{llll}
\hline Study & Mortality & Renal outcome & Short-term pulmonary outcome \\
\hline Biard et al. 2005 [9] & $2 / 20(10 \%)$ & RRT: 6/18 (33\%) survivors & 2 deaths due to pulmonary hypoplasia \\
Spaggiari et al. 2013 [11] & $2 / 11(18 \%)$ & Favorable in 3/9 survivors & N/A \\
Zaccara et al. 2005 [12] & $3 / 12(25 \%)$ & Creatinine in 9 survivors :1.3 $\pm 0.2 \mathrm{mg} / \mathrm{dl}$ after 1 year & 3 deaths due to pulmonary hypoplasia \\
Klaassen et al. 2007 [13] & $7 / 23(30 \%)$ & Neonatal RRT: 4/23 (17\%) & Mechanical ventilation: $14 / 23(61 \%)$ \\
Mehler et al. 2011 [14] & $13 / 36(36 \%)$ & Neonatal RRT: 7/23 (30\%) & Mechanical ventilation: 23/36 (64\%) \\
Spiro et al. 2015 [16] & $23 / 47(49 \%)$ & $n=14$ in follow-up, & N/A \\
Grijseels et al. 2011 [17] & $15 / 25(60 \%)$ & Neonatal ARF and/or later CKD: 4/13 (31\%) & Ventilation: $9 / 10(90 \%)$ survivors \\
Tomotaki et al. 2017 [18] & $21 / 28(75 \%)$ (no TOPs) & No RRT in survivors & All deaths due to pulmonary hypoplasia \\
Mehler et al. 2017 [19] & $6 / 38(16 \%)$ & Neonatal RRT: 12/35 (34\%) & Mechanical ventilation: 12/38 (32\%) \\
\hline
\end{tabular}

$A R F$ Acute renal failure, $C K D$ chronic kidney disease, N/A not applicable, $R R T$ renal replacement therapy, $T O P$ termination of pregnancy. 
Renal replacement therapy during the first 6 weeks of life was performed in around one-third of the children; thus $66 \%$ did not require dialysis initially [19]. These numbers are comparable (Table 1) with those reported in previous studies, and in some children discontinuation of dialysis is even possible [13]. It should be noted, however, that a proportion of patients not requiring dialysis in the neonatal period will ultimately develop CKD and need renal replacement therapy later in life.

In the Mehler study the mortality up to the time of hospital discharge was three of $38(8 \%)$ liveborn and actively treated children with a history of ROH; the overall mortality in this cohort rose to six (16\%; 3 children died after discharge) during the follow-up period of a median of 1.5 years. As stated above, the outcome was associated with the underlying cause of $\mathrm{ROH}$, even though the gestational age and bodyweight at birth did not differ. A better survival was observed for infants with a history of urinary tract obstruction (85\%) and ARPKD (71\%) compared with those with renal agenesis or kidney hypo -/dysplasia (30\%), a diagnosis that until recently was regarded as incompatible with life [19]. Thus, overall outcome seems to be even better compared to that reported in previous studies (Table 1), although the local policy regarding TOP might bias comparison of studies because fetuses with worse prognosis may be underrepresented. For example Tomotaki et al. report that none of the pregnancies evaluated in their study were terminated and that a mortality of $75 \%$ in liveborn children with a history of ROH was observed [18].

\section{The third way: Palliative care}

In addition to TOP or active postnatal treatment, Mehler et al. [19] describe a palliative treatment approach as an option (Fig. 1). In their study, $8 \%$ of the parents opted for palliative care after birth following prenatal counseling, and four additional patients chose this approach after initial active treatment. In total 11 of the 49 (22\%) liveborn children underwent palliative treatment [19]. In total, $45 \%$ (37\% TOP, $8 \%$ palliative care) of the counseled families opted primarily against continuation of the pregnancy or postnatal sustained medical treatment. This rate is comparable to that reported in other studies where only TOP was reported and chosen in 46$58 \%$ of the cases $[16,17]$.

\section{Practical consequences: counseling}

Counseling families with fetal bilateral renal disease and $\mathrm{ROH}$ is extremely challenging. As mentioned, it is impossible to exactly predict the postnatal course, as also underlined by
Fig. 1 Decision-making steps in the care of renal oligohydramnios (modified from [1], with permission). ROH Renal oligohydramnios

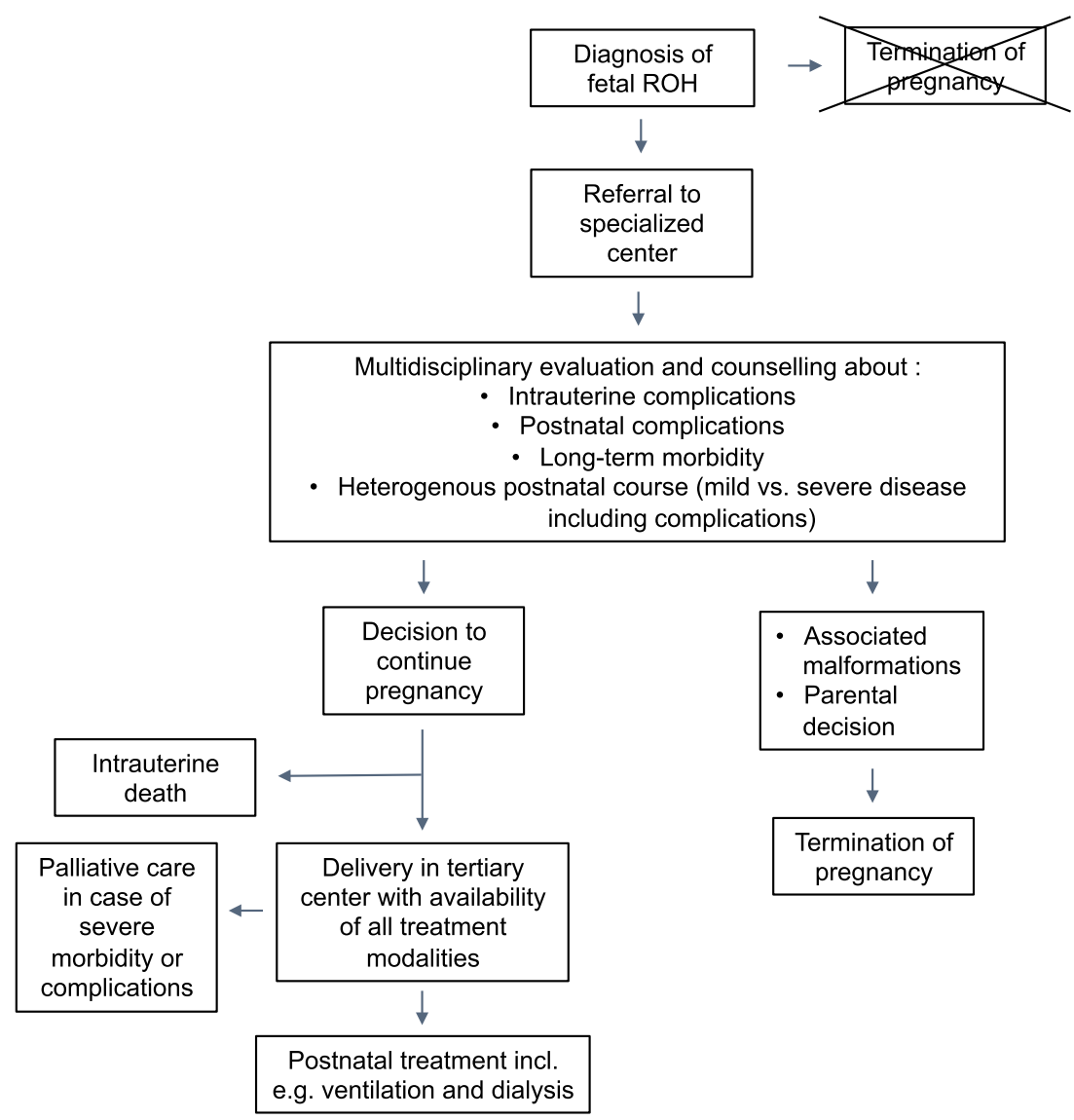


the current series of Mehler et al. [19], with outcome extremes ranging from fetal or neonatal death to mild long-term course or end-stage renal disease in childhood. Some families (and physicians) would only accept birth of a healthy child, and if this is the case, TOP seems a logical decision; others would accept birth of a child with chronic renal disease and would even accept additional complications.

In addition, there is a lack of consensus even among the medical specialities involved (obstetrics, neonatology, pediatric nephrology, urology, genetics and others). Obviously, the attitude towards treatment is also different between involved physicians, highlighted by the fact that in the current series $36.9 \%$ of pregnancies were terminated without multidisciplinary counseling. Unfortunately, the authors do not provide exact numbers on whether more TOP were performed without versus with multidisciplinary counseling; it would appear-as outlined in the discussion - that in the majority of families who opted for TOP, the parents only talked to the obstetricians. In this setting, the advances in neonatal intensive care, as well as pediatric nephrology, are not likely to be adequately discussed with families. In this respect, it is remarkable that the other extreme, i.e. decision for active antenatal treatment (e.g. amniocentesis in patients with lower urinary tract obstruction), is often also not discussed with pediatric nephrologists. Efforts need to be undertaken to resolve these discrepancies and establish standards.

This does not mean that TOP is not an option in the situation of $\mathrm{ROH}$ - rather that efforts to achieve an optimal, ethical decision-making process require informed counseling, including the discussion of all options. A valuable ethical framework for children with CKD has been suggested by Dionne and d'Agincourt-Canning [20], and parallel efforts to create a similar framework for $\mathrm{ROH}$ seems inevitable, especially since the current study and others have demonstrated that the outcome is not automatically fatal. Unfortunately, systematic studies providing other, equally important outcome parameters for families, such as quality of life, extrarenal morbidity, neuropsychological development, among others, are not available.

In this respect, the palliative approach described by Mehler et al. [19] also has important consequences on counseling as it provides the opportunity to delay the final decision for families who cannot decide immediately. TOP may not be acceptable for some families (e.g. for religious reasons). Palliative care is a way to minimize suffering without active treatment when severe morbidity and complications occur.

Taking all these points into consideration, as well as the data provided by Mehler et al. [19], we plead for a multidisciplinary approach, as is commonly practiced in other clinical situations, unless families do not wish it. In our personal view, in the twenty-first century, it seems hardly acceptable to accept unilateral counseling of families with $\mathrm{ROH}$ by obstetricians alone. Parents need to be adequately informed about the shortand long-term consequences of chronic renal disease but also about the progress that has been made. If possible, support from the psychosocial team should be sought, and discussions with families should be repeated, as is standard clinical practice in other decision-making situations.

\section{Conclusion}

Recent studies, including that of Mehler et al. [19] show that the outcome of children with a history of $\mathrm{ROH}$ is nowadays encouraging in the context of potential treatment options in neonatal intensive care units. The prognosis (mortality and morbidity) of actively treated children seems to be better than historically believed. Future studies should evaluate the longterm morbidity regarding $\mathrm{CKD}$, end-stage renal disease and extrarenal problems. These data will also have an important impact on counseling affected families in the future. A multidisciplinary team that includes obstetricians, neonatologists and pediatric nephrologists, not only in clinical care but also in the decision-making process, seems mandatory. The option of palliative care might be valuable for families and care givers.

\section{Compliance with ethical standards}

Conflict of interest The authors declare that they have no conflict of interest.

\section{References}

1. Aulbert W, Kemper MJ (2016) Severe antenatally diagnosed renal disorders: background, prognosis and practical approach. Pediatr Nephrol 31:563-574

2. Melo BF, Aguiar MB, Bouzada MC, Aguiar RL, Pereira AK, Paixao GM, Linhares MC, Valerio FC, Simoes ESAC, Oliveira EA (2012) Early risk factors for neonatal mortality in CAKUT: analysis of 524 affected newborns. Pediatr Nephrol 27:965-972

3. Magann EF, Ounpraseuth S, Chauhan SP, Ranganathan AS, Dajani NK, Bergstrom J, Morrison JC (2015) Correlation of ultrasound estimated with dye-determined or directly measured amniotic fluid volume revisited. Gynecol Obstet Investig 79:46-49

4. Tsatsaris V, Gagnadoux MF, Aubry MC, Gubler MC, Dumez Y, Dommergues M (2002) Prenatal diagnosis of bilateral isolated fetal hyperechogenic kidneys. Is it possible to predict long term outcome? BJOG 109:1388-1393

5. Morris RK, Malin GL, Khan KS, Kilby MD (2009) Antenatal ultrasound to predict postnatal renal function in congenital lower urinary tract obstruction: systematic review of test accuracy. BJOG 116:1290-1299

6. Gupta P, Kumar S, Sharma R, Gadodia A, Roy KK, Sharma JB (2010) The role of magnetic resonance imaging in fetal renal anomalies. Int J Gynecol Obstet 111:209-212 
7. Poutamo J, Vanninen R, Partanen K, Kirkinen P (2000) Diagnosing fetal urinary tract abnormalities: benefits of MRI compared to ultrasonography. Acta Obstet Gyn Scan 79:65-71

8. Kozinszky Z, Sikovanyecz J, Pasztor N (2014) Severe midtrimester oligohydramnios: treatment strategies. Curr Opin Obstet Gynecol 26:67-76

9. Biard JM, Johnson MP, Carr MC, Wilson RD, Hedrick HL, Pavlock C, Adzick NS (2005) Long-term outcomes in children treated by prenatal vesicoamniotic shunting for lower urinary tract obstruction. Obstet Gynecol 106:503-508

10. Morris RK, Malin GL, Quinlan-Jones E, Middleton LJ, Diwakar L, Hemming K, Burke D, Daniels J, Denny E, Barton P, Roberts TE, Khan KS, Deeks JJ, Kilby MD (2013) The percutaneous shunting in lower urinary tract obstruction (PLUTO) study and randomised controlled trial: evaluation of the effectiveness, cost-effectiveness and acceptability of percutaneous vesicoamniotic shunting for lower urinary tract obstruction. Health Technol Assess 17:1-232

11. Spaggiari E, Stirnemann JJ, Heidet L, Dreux S, Ville Y, Oury JF, Delezoide AL, Muller F (2013) Outcome following prenatal diagnosis of severe bilateral renal hypoplasia. Prenat Diagn 33:11671172

12. Zaccara A, Giorlandino C, Mobili L, Brizzi C, Bilancioni E, Capolupo I, Capitanucci ML, M DEG (2005) Amniotic fluid index and fetal bladder outlet obstruction. Do we really need more? J Urol 174:1657-1660

13. Klaassen I, Neuhaus TJ, Mueller-Wiefel DE, Kemper MJ (2007) Antenatal oligohydramnios of renal origin: long-term outcome. Nephrol Dial Transplant 22:432-439
14. Mehler K, Beck BB, Kaul I, Rahimi G, Hoppe B, Kribs A (2011) Respiratory and general outcome in neonates with renal oligohydramnios-a single-centre experience. Nephrol Dial Transplant 26:3514-3522

15. Hogan J, Dourthe ME, Blondiaux E, Jouannic JM, Garel C, Ulinski $\mathrm{T}$ (2012) Renal outcome in children with antenatal diagnosis of severe CAKUT. Pediatr Nephrol 27:497-502

16. Spiro JE, Konrad M, Rieger-Fackeldey E, Masjosthusmann K, Amler S, Klockenbusch W, Schmitz R (2015) Renal oligo- and anhydramnios: cause, course and outcome - a single-center study. Arch Gynecol Obstet 292:327-336

17. Grijseels EW, van Hornstra PT, Govaerts LC, Cohen-Overbeek TE, de Krijger RR, Smit BJ, Cransberg K (2011) Outcome of pregnancies complicated by oligohydramnios or anhydramnios of renal origin. Prenat Diagn 31:1039-1045

18. Tomotaki S, Toyoshima K, Shimokaze T, Shibasaki J, Nagafuchi H (2017) Association between cord blood cystatin C levels and early mortality of neonates with congenital abnormalities of the kidney and urinary tract: a single-center, retrospective cohort study. Pediatr Nephrol 32:2089-2095

19. Mehler K, Gottschalk I, Burgmaier K, Volland R, Buescher AK, Feldkötter M, Keller T, Weber LT, Kribs A, Habbig S (2017) Prenatal parental decision-making and postnatal outcome in renal oligohydramnios. Pediatr Nephrol. https://doi.org/10.1007/s00467017-3735-Z

20. Dionne JM, d'Agincourt-Canning L (2015) Sustaining life or prolonging dying? Appropriate choice of conservative care for children in end-stage renal disease: an ethical framework. Pediatr Nephrol 30:1761-1769 